\title{
A strong micropillar containing a low angle grain boundary
}

\author{
R. Maaß, S. Van Petegem, D. Grolimund, and H. Van Swygenhoven ${ }^{\text {a) }}$ \\ ASQ/NUM Materials Science and Simulations, Paul Scherrer Institut, CH-5232 Villigen PSI, Switzerland
}

\author{
M. D. Uchic \\ Air Force Research Laboratory, Materials \& Manufacturing Directorate, Wright-Patterson AFB, \\ Ohio 45433-7734, USA
}

(Received 23 July 2007; accepted 22 August 2007; published online 28 September 2007)

\begin{abstract}
In situ white beam Laue diffraction experiments on focused ion beam machined single crystal $10 \mu \mathrm{m} \mathrm{Ni}$ pillars are performed to explore the relation between the initial and evolving microstructures and the subsequent flow response. The pillar has a flow stress that is at least in the upper bound of the scatter of the flow stresses obtained for similar Ni pillars. Detailed analysis of the Laue pattern suggests that the strength is in part due to the low angle grain boundary acting as a dislocation barrier. (c) 2007 American Institute of Physics. [DOI: 10.1063/1.2784938]
\end{abstract}

The strength of small single crystalline metallic pillars with diameters in the micron or even submicron regime is considerably higher than what is obtained for large samples. ${ }^{1-7}$ Stress-strain curves derived from microcompression tests performed on pillars synthesized using focused ion beam (FIB) milling demonstrate an increase in strength for smaller pillar diameters. Using ex situ and in situ Laue diffractions it has been shown that pillars made by FIB have preexisting strain gradients and that these can play an important role in the activation of slip systems during compression. ${ }^{8}$ Time resolved white beam Laue diffraction performed on $2 \mu \mathrm{m}$ diameter $\mathrm{Au}$ pillars demonstrated that plasticity starts on a hard slip system that is geometrically not predicted, but selected because of the character of the preexisting strain gradient and that in contrast to what was assumed, crystal rotation occurs during compression, ${ }^{9}$ which later also has been confirmed by FEM simulations ${ }^{10}$ and TEM studies. ${ }^{11}$ A similar study on a $10 \mu \mathrm{m}$ Au pillar synthesized by the same method, demonstrated slip starting on the classical geometrical predicted slip system involving unconstrained rotation of the crystal with respect to the compression axis. Such results suggest that the relationship between strength and the pillar size is complex and that microstructural details have to be included. This is supported by the recent observation that bcc Mo pillars with a diameter range of $0.3-1 \mu \mathrm{m}$ that are prepared using a chemical etch exhibit high strengths approaching the theoretical shear strength without any sign for a dependence on the pillar diameter. ${ }^{12}$

Besides the increase in flow stress for decreasing pillar diameter, another hallmark of size-affected flow in FIBprepared fcc micropillars is a relatively large scatter of flow strength for similar pillar sizes synthesized from the same bulk material. For instance, the shear flow stress of a $10 \mu \mathrm{m}$ Ni pillar in the study by Dimiduk et al. was observed to lie between 25 and $55 \mathrm{MPa}$, which is generally higher than the $\sim 20 \mathrm{MPa}$ for the bulk crystal used in that study. ${ }^{2}$ The fact that those flow-stress values overlapped with data obtained for 4-6 $\mu \mathrm{m}$ pillars $(30-70 \mathrm{MPa})$ supports the idea that the internal structure can play an important role in the scaledependent response. To address this issue, this letter reports an in situ Laue study performed on two $10 \mu \mathrm{m}$ Ni pillars that

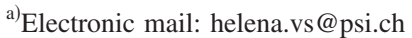

have been fabricated using a slightly modified FIB procedure based as the one reported in Ref. 13, and subsequently characterized using the methodology reported in Ref. 9. The modification in the synthesis procedure consisted of preparing a thin film from the bulk crystal using mechanical polishing, which was then mounted in the geometry required for in situ Laue. ${ }^{9}$ The diffraction studies demonstrate that the considered pillars contain a preexisting strain gradient (excess dislocation density) on the slip plane with the highest Schmid factor. Nevertheless, upon deformation both pillars turned out to have a very high flow stress. The details and the dynamics of the Laue diffraction, however, show the presence of a preexisting low angle grain boundary (GB) transecting the entire pillar, suggesting that for the pillars studied in the current work, the strength is influenced by the presence of the grain boundary.

Two single crystal Ni pillars (labeled A and B) with a diameter of $10 \mu \mathrm{m}$ and an aspect ratio of $3: 1$ prepared using the annular FIB milling procedure ${ }^{13}$ are deformed in situ at the MicroXAS beamline of the Swiss Light Source using a microcompression device equipped with a Hysitron Triboscope $(C)$ one-dimensional transducer ${ }^{9}$ allowing for a maximum force of $10 \mathrm{mN}$. The compression axis of the pillars roughly correspond with an $\sim[068]$ crystal axis and the incoming beam with an $\sim[93-2]$ axis. The samples A and B were compressed in situ with a loading rate of 1.5 and $3 \mu \mathrm{N} \mathrm{s}^{-1}$ respectively. The samples turned out to be very strong so that the technically imposed maximum force of the transducer was already reached at a total strain of $0.2 \%$ for pillar A and $0.3 \%$ for pillar B. Figure 1 shows the engineering stress strain curves together with the data (black curves) taken from Ref. 2 obtained for Ni pillars made by FIB from the same original $\mathrm{Ni}$ single crystal but with a compression axis corresponding to a [269] crystal axis. The black curves demonstrate the large scattering obtained for similar pillars. Pillars A and B seem to have a flow stress that is higher or within the upper bounds of the scatter for the [269] pillars.

Prior to loading the pillars were scanned with a focused white x-ray beam, the full width of half maximum of the beam being $2.2 \mu \mathrm{m}$. Laue diffraction patterns were recorded with a scan step of $2 \mu \mathrm{m}$ in the vertical direction. In the horizontal direction the central axis and the outer axis, distanced $\pm 3 \mu \mathrm{m}$ from the center, were scanned. Each Laue 


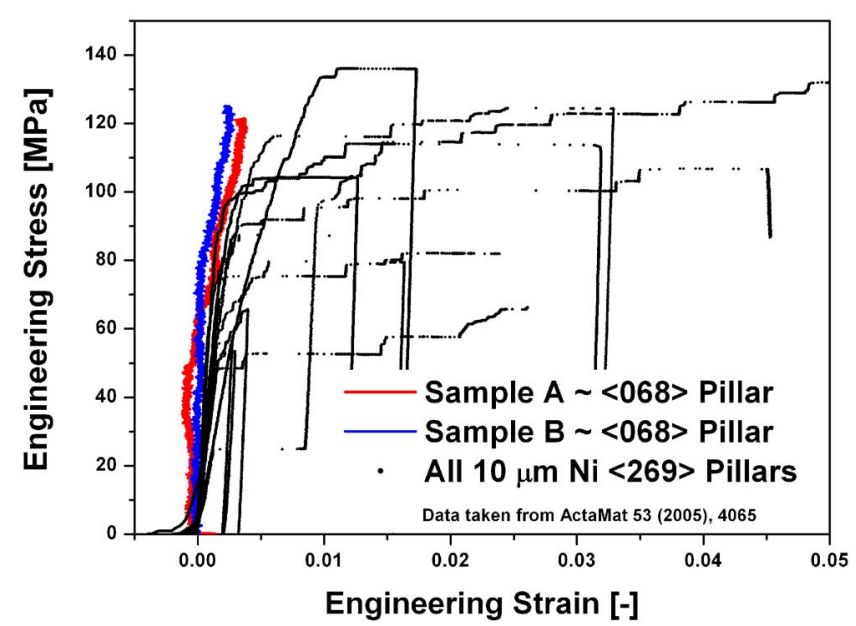

FIG. 1. (Color online) Stress strain data of in situ deformed Ni pillars A and $\mathrm{B}$ and ex situ deformed Ni pillars taken from Ref. 2.

pattern taken includes the $(0-20),(002),(11-1),(0-22)$, $(33-1)$, and $(31-3)$ diffraction peaks. Figure 2(a) displays for pillar A the spatially resolved $(0-20)$ reflection over the entire pillar and Fig. 2(b) shows the scanning electron microscopy (SEM) picture of the pillar prior to deformation. For all Laue reflections a shift in the peak position can be observed when scanning along the pillar axis indicating crys-

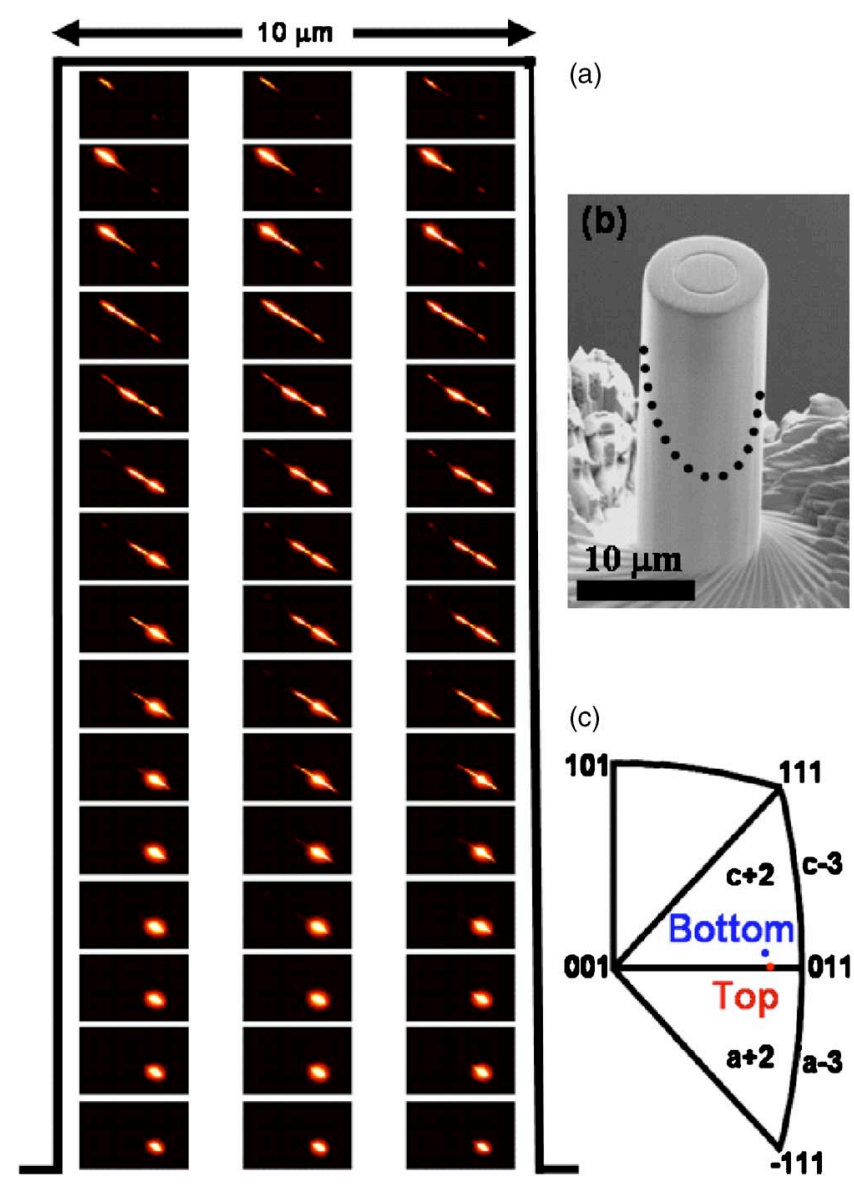

FIG. 2. (Color online) (a) (0-20) Bragg reflection map of the undeformed $\mathrm{Ni}$ - pillar A-the black line indicates the outer contour of the pillar. (b) SEM image of the same pillar with the approximate location of the transecting boundary plane (dashed line). (c) The standard stereographic triangle showing the classical predicted slip systems and the orientation of the compression axis at the bottom and the top of the pillar.
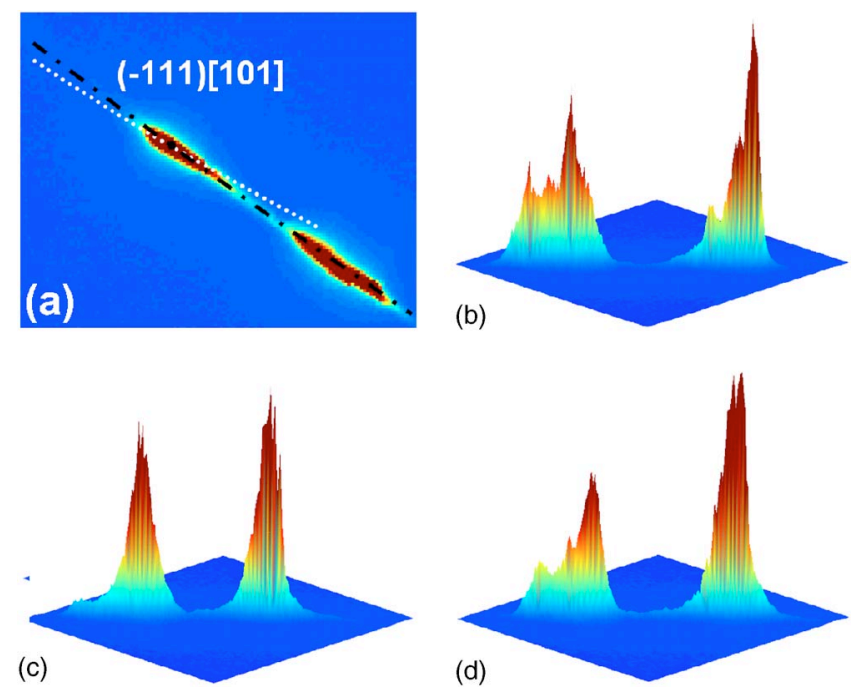

FIG. 3. (Color online) (0-20) Laue spot prior to deformation. (a) 2D image, the white line is the streaking direction considering only tilt, and black with tilt and twist components, (b) 3D image (linear scale), (c) during deformation and (d) after unloading. All peaks were recorded $10 \mathrm{~mm}$ below the pillar top surface and on the central axis.

tal rotation. In the direction perpendicular to the pillar axis, only minor changes among the Laue patterns can be distinguished. In the central part of the pillar, all Laue spots are split and evidence pronounced streaking, some more extended than others. In other words, the upper and lower parts of the pillar have a slightly different orientation, the splitting of the peaks demonstrating the existence of a low angle grain boundary. Using the position of all Laue spots, the exact orientation of the compression axis can be calculated for the top and the bottom of the pillar. Figure 2(c) shows the position of the compression axis in the standard stereographic triangle prior to deformation for the bottom of the pillar (blue) corresponding with a [5 60 80] axis and for the top of the pillar (red) corresponding with a [ [ 161 79] axis. The misorientation of the compression axis between the top and bottom of the pillar is about $2.5^{\circ}$.

Two of the most intense Laue spots (0-20) and (002) exhibit the largest streaking. Assuming an excess number of pure edge dislocations ${ }^{14,15}$ the best correspondence between theory and experimentally observed streaking and splitting correspond to the $a-3(111)[-110]$ or $c+2(-111)[101]$ system. The fitting is, however, not perfect, as shown on Fig. $3(\mathrm{a})$, where the $(0-20)$ spot is pictured together with the streaking direction for the $c+2$ system (white dashed line). Careful inspection of all other Laue spots, taking into account that the length of the streaking is determined by the contrast factor and the projection on the detector plane, demonstrates that the $a-3$ system can be excluded because it does not correspond with the streaking observed in the other diffraction spots. For instance, the $a-3$ streaking direction is nearly perpendicular to the streaking direction observed for the $(31-3)$ spot. Note that $c+2$ is the primary slip system for the orientation of the compression axis at the bottom of the pillar. The fact that the fitting is, however, not perfect, suggests that the grain boundary cannot be described as a pure tilt boundary around the $[-1-21]$ axis. By adding a twist component, i.e., a rotation around the [101] axis the fitting improved for all measured diffraction peaks. The black dashed line in Fig. 3(a) shows the expected direction for 

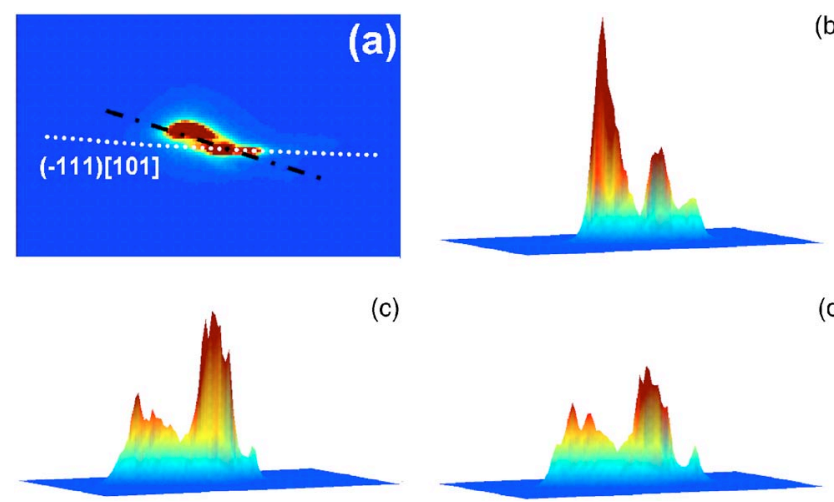

(c)

FIG. 4. (Color online) (11-1) Laue spot prior to deformation. (a) 2D image, the white line is the streaking direction considering only tilt, and black with tilt and twist components. (b) 3D image (linear scale), (c) during deformation and (d) after unloading. All peaks were recorded $10 \mathrm{~mm}$ below the pillar top surface and on the central axis.

peak splitting for the $(0-20)$ taking into account a $3.2^{\circ}$ tilt component around the $[-1-21]$ axis and a $0.5^{\circ}$ twist component around the [101] axis. Figure 2(b) shows on the SEM picture a schematic presentation of the grain boundary plane relative to the compression axis and the beam direction.

Figures 3 and 4 show, respectively, the $(0-20)$ and the $(11-1)$ Laue spot before compression [(a) in two dimensions (2D) and (b) in three dimensions (3D)], (c) during compression, and (d) after loading measured at $\sim 10 \mathrm{~mm}$ below the pillar top. During compression the $c+2$ streaking observed in both parts of the $(0-20)$ spot seems to reduce, and the peaks do not return to their original form upon unloading. Also for the $(11-1)$ spot the streaking and splitting can be assigned to the $c+2$ system after the correction for the twist (black line) has been performed. The splitting of the peak is much less pronounced. During deformation the peak width in the streaking direction is about constant but there are variations in intensity distribution. The differences between $(0-20)$ and (11-1) spots upon loading can be explained in terms of geometrical considerations. The normal of the $(11-1)$ plane makes a small angle with the tilt axis. As a result, this diffraction spot is less affected by the tilt component. On the other hand, the $(0-20)$ planes make a large angle with the tilt axis and are therefore sensitive to the tilt component, which is reflected in the larger splitting of this peak compared to the $(11-1)$ peak. It is worth noting that the changes in the peak profile and the intensity spread after unloading are only occurring in the upper part of the pillar, whereas in the lower part (the first $8 \mu \mathrm{m}$ corresponding with the first four Laue spots when starting from the bottom of the pillar in Fig. 1) does not exhibit any changes. This suggests that dislocation activity is influenced by the presence of the GB.

The above detailed analysis prior and during in situ deformation of pillar A demonstrates the presence of a low angle grain boundary transecting the entire pillar, having a twist and a tilt component. The high observed strength of the pillar during the initial loading may be in part due to a grain boundary acting as a dislocation barrier. It is worth noting that the analysis of pillar B is qualitatively similar, in the sense that also this pillar contains a low angle grain boundary. The GB plane in pillar B is at about the same position, but, however, has slightly different values for twist and tilt reflected in a total misorientation of $3.2^{\circ}$ compared to the $2.5^{\circ}$ of pillar A.

In summary, the in situ Laue study performed allowed to identify the presence of a low angle grain boundary with a tilt and a twist component in $10 \mu \mathrm{m}$ FIB Ni pillars. These pillars were observed to have a strength higher or within the upper bounds of previously published values. How this GB was formed, whether it is a result of the FIB procedure or it was introduced, for instance, by handling the sample is unknown. Current results urge for the systematic performance of in situ Laue diffraction experiments, allowing for a direct correlation between the microstructure and the strength on the pillar diameter. Such an approach will reduce the large scatter observed for similar pillar diameters since those results issuing from different initial microstructures will no longer belong to the domain of the statistically unknown.

H.V.S. thanks the European commission for financial support of the NANOMESO (FP6). The authors also thank C. Borca and M. Willimann from the MicroXas/SLS for their technical support. Further gratitude is expressed to Dr. U. Hangen and M. Berg from Hysitron Incorporated for supporting the TriboScope $\odot$ implementation.

${ }^{1}$ M. D. Uchic, D. M. Dimiduk, J. N. Florando, and W. D. Nix, Science 305, 986 (2004).

${ }^{2}$ D. M. Dimiduk, M. D. Uchic, and T. A. Parthasarathy, Acta Mater. 53, 4065 (2005).

${ }^{3}$ J. R. Greer, W. C. Oliver, and W. D. Nix, Acta Mater. 53, 1821 (2005).

${ }^{4}$ J. R. Greer, W. C. Oliver, and W. D. Nix, Acta Mater. 54, 1705 (2006).

${ }^{5}$ J. R. Greer and W. D. Nix, Phys. Rev. B 73, 245410 (2006).

${ }^{6}$ C. A. Volkert and E. T. Lilleodden, Philos. Mag. 86, 5567 (2006).

${ }^{7}$ C. A. Volkert and E. T. Lilleodden, Appl. Phys. Lett. 89, 061920 (2006).

${ }^{8}$ R. Maaß, D. Grolimund, S. Van Petegem, M. Willimann, M. Jensen, H. Van Swygenhoven, T. Lehnert, M. A. M. Gijs, C. A. Volkert, E. T. Lilleodden, and R. Schwaiger, Appl. Phys. Lett. 89, 151905 (2006).

${ }^{9}$ R. Maaß, S. Van Petegem, H. Van Swygenhoven, P. M. Derlet, C. A. Volkert, and D. Grolimund, "Time resolved Laue diffraction of deforming micropillars," Phys. Rev. Lett. (in press).

${ }^{10}$ D. Raabe, D. Ma, and F. Roters, Acta Mater. 55, 4567 (2007).

${ }^{11}$ C. P. Frick, B. G. Clark, S. Orso, and E. Arzt, "Size effect on strength and strain hardening of [111] Nickel sub-micron compression pillars," Acta Mater. (submitted)

${ }^{12}$ H. Bei, S. Shim, E. P. George, M. K. Miller, E. G. Herbert, and G. M. Pharr, Scr. Mater. 57, 397 (2007).

${ }^{13}$ M. D. Uchic and D. A. Dimiduk, Mater. Sci. Eng., A 400-401, 268 (2005).

${ }^{14}$ R. I. Barabash, G. E. Ice, and F. J. Walker, J. Appl. Phys. 93, 1457 (2003).

${ }^{15}$ R. I. Barabash and G. E. Ice, Encyclopedia of Materials: Science and Technology Updates (Elsevier, Oxford, UK, 2005), p. 1. 